\title{
Semi-closed-circuit vacuum-assisted mini percutaneous nephrolithotomy in the pediatric population: the initial experience of two tertiary referral centers
}

Journal: Minerva Urologica e Nefrologica

Paper code: Minerva Urol Nefrol-3951

Submission date: May 15, 2020

Article type: Original article

Files:

1. Reply letter to comments on the manuscript

Version: 1

Description:

File format: application/vnd.openxmlformats-officedocument.wordprocessingml.document

2. Manuscript

Version: 2

Description: Revised manuscript - Gallioli

File format: application/vnd.openxmlformats-officedocument.wordprocessingml.document

3. Figures 1

Version: 1

Description: Figure 1 - Gallioli

File format: image/tiff

4. Figures 2

Version: 1

Description: Figure 2 - Gallioli

File format: image/tiff

5. Supplementary Digital Material 1

Version: 1

Description: Supplementary file - Gallioli

File format: application/vnd.openxmlformats-officedocument.wordprocessingml.document 


\section{REVISION NOTES}

Dear Editor, please find enclosed the revised version of the manuscript entitled "Semi-closed-circuit vacuumassisted mini percutaneous nephrolithotomy in pediatric patients" (Authors: Gallioli et al.; Minerva Urol Nefrol-3951) to be considered for publication in the Minerva Urologica \& Nefrologica.

We are very grateful to the Reviewers and the Editors for their insightful comments to our paper.

Below, please find item-by-item responses to the comments, which are included rerbatim. All pages and paragraph numbers refer to locations in the revised manuscript:

List of the changes made in the manuscript:

\section{Reviewer 1:}

Comments to the Author In the current study, Gallioli et al. reported their initial experience on the use of a vacuum device for mini-PCNL in paediatric patients. The analysis was based on 18 mini-PCIIs performed in 13 children. Absence of stone fragments more than $4 \mathrm{~mm}$ (stone free rate - SFRD) and cost analysis were the primary outcomes of the study. Interesting $81.3 \%$ SFR was recorded in absence of peri and postoperative complications.

Overall, the manuscript sounds scientifically well.

However, it should be considered that the study is a case-senge carried out in two high-volume urological hospitals. The nature of the strady was the main limit as like authors have recognized too.

We are grateful to Reviewer 1 for the important and generally positive comments to our paper.

Major corrections:

1. As previously underlined, the study sample is underpower to analyse "safety and efficacy" as well it was not comparative. Thus, discussion sentences on "safety and efficacy should be replaced by "feasibility" idea.

\section{Answer 1:}

We agree with Reviewer 1 that the study is underpowered to analyze safety and efficacy of the vacuum device for mini-PCNLin the pediatric population. However, the study demonstrated that the device is safe, as no complications were reported during the procedures and the only postoperative major complication was represented by stent positioning for hydronephrosis and fever. Therefore, the concept of "safety and efficacy" was replaced by "safety and feasibility".

Minor corrections:

2. postoperative CT has not been performed. That limit has been recognized in discussion, however underline that SFR may be overestimated due to absence of a more accurate imaging exam.

\section{Answer 2:}

We agree with the Reviewer 1 . Therefore, a sentence underlining the risk of SFR overestimation was added in the Discussion section.

3. Then, have you considered to include experimental studies (if published) recording intra renal pressure by using ClearPetra system? This may improve your rationale based on mathematical count (Bernoulli' principle) descripted in methods.

\section{Answer 3:}

We thank the Reviewer for this insightful comment. A study from Zanetti et al. (Vacuum-assisted mini-percutaneous nephrolithotomy: a new perspective in fragments clearance and intrarenal pressure 


\section{Reviewer 2:}

Comments to the Author

The joint effort has to be appreciated. The device is of interest but the results have to be critically interpreted and extensively discussed, while the final conclusions should be cautious, taking into serious account that we are talking of children (8-13 year-old).

We are grateful to Reviewer 2 for the important comments io our paper.

Major corrections:

1. A procedure in a child lasting 2 hours under general anesthesia, sometimes requiring 2 tracts, after about $50 \%$ of preoperative procedures like $\mathbf{H}$, nephrostomy or both, with a $28 \%$ of postoperative fever (cut only $5.6 \%$ prep positive urine culure), a $39 \%$ of complications up to Clavien Dindo IIIa and the need for a further PNL or RIRS ino $2 \%$ of cases is for sure feasible but not really miniinvasive as in the intentions of a miniaturized approach. Absence of blood transfusions is an issue but not the only one.

\section{Answer 1:}

We completely agree with Reviewer 2 . The pediatric population that has been analyzed in the present study is generally complex, requiring a complex approach to treat the stones. The complications were mainly represented by fever ( $5 / 7$ complications) which risk factors are represented by staghorn calculi (44\% in our patients), low patient age and positive preoperative urine culture (Gutierrez J, Smith A, Geavlete $\mathrm{P}$, et al. Urinary tract infections and post-operative fever in percutaneous nephrolithotomy. World J Urol. 2013;31(5):1135-1140. doi:10.1007/s00345-012-0836-y). So, our population had a high risk of infectious complications even if the preoperative urine culture was positive in only $5.6 \%$ patients. Moreover, the only high-grade complication was represented by stent DJ placement for hydronephrosis and fever. Therefore, we believe that the present treatment was as mini-invasive as possible in this specific population.

2. The choice of a rigid-only procedure of this kind with a number of technical limitations, especially in larger/staghorn stones (and often in metabolic young patients, should be extensively discussed. One should know why to choose this kind of procedure rather than a bit larger tract access with the possibility of quicker non-laser lithotripsy, better irrigation outflow, higher SFRs and may be lower complication rates. 


\section{Answer 2:}

We thank Reviewer 2 for this important issue that needs to be clarified. The procedure was rigid but, in case of suspect of residual fragments, a flexible nephroscopy was performed.

The miniaturization of the technique may be particularly beneficial in the pediatric population, which in our case had a median age of 9 years and a median weight of $29 \mathrm{~kg}$. It has been demonstrated that the renal injury following dilation is significantly wider when the tract is more than 22 French (Emiliani E, Talso M, Baghdadi M, Traxer O. Renal parenchyma injury after percutaneous nephrolithotomy tract dilatations in pig and cadaveric kidney models. Cent European J Urol. 2017;70(1):69-75. doi:10.5173/ceju.2017.930). A tract dilation of 16 French generates a renal fissure of a diameter of $4.4 / 6.28 \mathrm{~mm}$ in cadaveric/porcine model. On the other hand, a tract dilation of 24 French (standard-PCNL size) generates a renal fissure of $7.49 / 12.53 \mathrm{~mm}$, almost doubling the diameter of a mini-PCNL and tripling the total dilation area. These findings explain why the tract dilation size is associated with hemorrhagic complications. The impact of a tract dilation on a pediatric kidney, which mean longest diameter is $9 \mathrm{~cm}$ in 10-year-old patients, is even more significant. Moreover, a staghorn stone may require more than one aecess, independently from the size of dilation, to reach and treat efficiently all the stones. For these reasons, we chrse to perform mini-PCNL with 14 and 16 French access sheaths in these patients.

We acknowledge that the surgical time was generally long for the procedure Hever, the total operative time did not reflect the total time spent in the renal cavity. Our surgical times might be affected by the first steps of the procedure as cystoscopy and ureteral eatheterization (which might be challenging in Valdivia-Galdakao position) are performed by naivesesidents under the guidance of a tutor. We do not exceed 90 minutes of effective PCNI-surgery 10 reduce the risk of infectious complications. The irrigation outflow was guaranteed by the use of the ClearPetra system, which recently demonstrated to maintain mean intrarenal pressures of $15 \mathrm{cmH}_{2} \mathrm{O}(11.03 \mathrm{mmHg})$ during mini-PCNL (Zanetti et al. Vacuum-assisted mini-percutanegos nephrolithotomy: a new perspective in fragments clearance and intrarenal pressure contrô. World J Urol. 2020;10.1007/s00345-02003318-5. doi:10.1007/s00345-025-03318-5) and allows an optimal visualization of the intrarenal cavity. Finally, the stone-free rate and the complication rate were in line with current literature.

3. Costs should include prolonged hospitalizations for complications and ancillary procedures, not only of the single accessories! A honest discussion of such data is desirable, although the economic aspect is not a priopity, compared to the pure clinical aspects.

\section{Answer 3:}

We agree with Reviewer 2. A conpetete cost analysis should also include complications and ancillary procedures. Considering that our study has no control group as reference, we could not compare complieations and ancillary procedures to a standard procedure. The aim of our analysis of materials costs was to show the sustainability of the ClearPetra, which is disposable, in comparison to re-usable device that is currently employed in our Institutions (MIP Storz). Therefore, we changed the title of the dedicated paragraph, as the title "cost analysis" might be misleading.

Minor corrections:

4. Abstract, Methods: SFR was defined ... please specify with which kind of imaging (also lacking in the Materials and methods), to be added.

\section{Answer 4:}

The definition of stone-free rate and imaging performed during follow-up was added in Methods and Abstract.

5. Abstract, Results: the costs... please specify that costs do not include hospital stay but just instruments and devices.

\section{Answer 5:}

The sentence was corrected, specifying that the materials costs were only analyzed. 
6. Abstract, Results: what is standard mini-PNL for the authors in children? Please add.

\section{Answer 6:}

The sentence was corrected, and the term "standard mini-PCNL" was substituted by "mini-PCNL using a reusable set”.

7. Throughout the text references are sometimes after the punctuation and not before. Please check according to the instructions of the authors, also for how to cite multiplmaginablee references.

\section{Answer 7:}

The references in the text were completely checked and the errors in punctuation and citation style were corrected.

8. $12 \mathrm{~F}$ nephroscope, which one? size of the working channel? $9.5 \mathrm{~F}$ semirigid ureteroscope, which one? size of the working channel. Please compare the irrigation outflow in two different devices like this. What about the length of the ureteroscope in a child and in percutaneous nephrolithotomy? please discuss.

\section{Answer 8:}

We used a 12 Fr nephroscope (Karl Storz SE \& Co. KG, Germany; length: 22 m; 6.7 Fr) for the 16 Fr ClearPetra, and a 9.5 Fr semirigid ureteroscope (Kani Storz SE \& Co. KG, Germany; length: $34 \mathrm{~cm}$; working channel $5 \mathrm{Fr}$ ) was ysed for the $14 \mathrm{Fr}$ (ciearPetra. The operators were used to $9.5 \mathrm{Fr}$ semirigid ureteroscope in the setting of min-PCNL and felt comfortable with 34 $\mathrm{cm}$ length (the shorter of the Storz ureteroscopes on the market).

9. 1-5J x 10-20 Hz laser settings, please discuss the risk of heating within the collecting system with a 2 hours-procedure.

\section{Answer 9:}

We thank the Reviewer for this crucial comment. We corrected a typing error in the manuscript as our settings are 1-1.5J x 10-20 Hz.

10. the kind of anesthesia is not reported, although imaginable.

Answer 10:

The type of anesthesia (general) was reported.

Editorial revision.

Manuscript: The supplementary material has been quoted in the text.

Bibliography: the DOis from the references were removed.

We thank the Editor-in-Chief for the overall positive comment to our paper. The text has been revised accordingly.

We hope that the paper is now suitable to be considered for publication in the Minerva Urologica \& Nefrologica.

Sincerely yours,

Andrea Gallioli on behalf of all the authors 
Semi-closed-circuit vacuum-assisted mini percutaneous nephrolithotomy in the pediatric population: the initial experience of two tertiary referral centers

Andrea Gallioli ${ }^{*}$, Alfredo Berrettini ${ }^{2}$, Gianluca Sampogna ${ }^{1,2}$, Erika Llorens ${ }^{3}$, Yesica Quiróz ${ }^{3}$, Michele Gnech ${ }^{2}$, Elisa De Lorenzis ${ }^{5}$, Giancarlo Albo ${ }^{5}$, Joan Palou ${ }^{4}$, Gianantonio Manzoni², Anna Bujons $^{3}$, Emanuele Montanari ${ }^{5}$

${ }^{1}$ Urology Unit, Fondazione IRCCS Ca' Granda Ospedale Maggiore Policlinice, University of Milan, Milan, Italy

2 Pediatric Urology Unit, Fondazione IRCCS Ca' Granda Ospedale Maggiore Policlifice, Milan, Italy

${ }^{3}$ Pediatric Urology Division, Fundaciò Puigvert, Barcelona, Spain

${ }^{4}$ Urology Department, Fundació Puigvert, Barcelona, Spain

${ }^{5}$ Urology Unit, Fondazione IRCCS Ca' Granda Ospedale Maggiore Policlinico, Department of Clinical Sciences and Community Health, University of Milan, Diglan, Italy

*Corresponding author: Andrea Gallioli, MD

Urology Unit, Fondazione IRCES Ca' Granda Ospedale Maggiore Policlinico.

Via della Commenda 15.

Milan 20122, Italy.

Tel. +393496645072

E-mail address: andrea.gallioli@gipal.com

Keywords: percutaneous nephrolithotomy; urolithiasis; litholapaxy; children; kidney calculi 


\section{ABSTRACT}

\section{Background}

Percutaneous nephrolithotomy (PCNL) is the gold-standard for complex renal stones treatment in the pediatric population. While the miniaturization of PCNL reduces the risk of bleeding, it can hinder surgical and functional outcomes. The aim of the study is to assess the safety and feasibility of semiclosed-circuit vacuum-assisted Mini-PCNL (vmPCNL) in pediatric patients.

\section{Methods}

From January 2017 to December 2018, we prospectively collected data on consecutive vmPCNLs from two European tertiary referral centers. The procedure was performed with the $e^{A}$ earPetra® access sheath equipped with a lateral arm connected to the aspiration system (pressure setting 120$150 \mathrm{cmH}_{2} \mathrm{O}$ ) by a $200 \mathrm{ml}$ plastic stone collector. Pre-, intra- and post-operative data and costs were analyzed. The stone-free rate (SFR) was defined as absence of residual fragments $>4 \mathrm{~mm}$ with either ultrasound or kidney, ureter, and bladder x-ray.

\section{Results}

Eighteen vmPCNLs were performed in 16 renal units of 13 children. The median age was 119 months (IQR: 97-160) and the weight was 20 Kg (IQR: 25040$)$. The median cumulative stone size was 32 mm (22-46) with 8 (44.4\%) cases of staghorn stones. The OT was 128 min (IQR: 99-167). The basketing was unnecessary in 6/18 (33\%) cases. Neither intra-operative complications nor blood transfusions occurred. Post-operative fever was observed in 5/18 (27.8\%) cases; in one case a double J ureteral stent was placed for coneomitant hydronephrosis. The SFR was $81.3 \%(13 / 16)$, rising to 93.8\% (15/15) after anciliary procedures. The materials costs of a vmPCNL (734.8 €) were comparable to mini-PCNL using a reusable set $(710.7 €)$.

\section{Conclusions}

The vmPCNL seems to be sustainable, safe and feasible for kidney stones treatment in the pediatric population. 


\section{INTRODUCTION}

Surgical treatment of pediatric kidney stones has changed over the years [1]. Percutaneous nephrolithotomy (PCNL) was firstly introduced in 1976 and it still has a leading role despite many technological advancements in retrograde approaches [2-4]. According to the EAU guidelines, PCNL remains the first surgical option for renal stones $>20 \mathrm{~mm}\left(\sim 300 \mathrm{~mm}^{2}\right)$, for lower pole cáliceal stones $>10 \mathrm{~mm}$ and for staghorn or multiple stones [5]. It ensures a higher stone-free rate (SFR) than shockwave lithotripsy (SWL) and retrograde intra-renal surgery (RIRS), despice presenting a higher rate of major complications and a higher decrease of post-operative hemogløbin levels [6]. In order to reduce PCNL morbidity, Jackman et al. introduced the Mini-PCNL in 1998, reporting encouraging results in terms of complication rate reduction. Their findings were subsequently confirmed by several randomized controlled trials (RCTs) [7. Although mini-PCDIt (nephrostomy tract size $<20 \mathrm{Fr}$ ) may reduce the number of major complications (haemorrhage in particular), some authors argue that it may decrease the SFR, lengthen the operative time (OT, and increase the number of post-operative urinary tract infections (UTI) [8,9].

Endourological research is currently focusing on regulating intra-renal pressures, especially, to reduce the risk of infection. During surgery, irrigation flow and irrigation pressures tend to be increased in order to achieve better visibility $\{10]$. Increased intrarenal pressures can provoke the deterioration of the renal parenchyma, and causepyelorenal backflow, fluid reabsorption, and bacteremia [11]. Novel systems to control and timit the increase of intra-renal pressures, like a semiclosed-circuit vacuum-assisted mini-PCNL (rmPCNL) system, may potentially reduce the number of post-operative compications and overcome some of the above-mentioned limitations of a minimally in asive approaches [12?.

The aim of the strdy is to report our initial experience using a vmPCNL system for renal stone treatment and to assess its safety and efficacy in pediatric patients.

\section{MATERIALS AND METHODS}

We prospectively collected data from two European tertiary referral centers: Fondazione IRCCS Ca' Granda - Ospedale Maggiore Policlinico (Milan) and Fundació Puigvert (Barcelona). From January 2017 to December 2018, we enrolled to the study all consecutive < 18 years old at the time of surgery undergoing a vmPCNL. Patients' data and surgical outcomes were retrospectively analyzed. The study was conducted in accordance with the Declaration of Helsinki (1964) and its later amendments. The study was approved by the Institutional Review Board. All patients signed an informed consent at the time of hospitalization to share anonymously clinical information for research purposes. 


\section{Data collection}

Pre-operative patient data included demographic variables (e.g. age, sex), medical history and stone characteristics, like cumulative stone size (evaluated as sum of largest stones diameter) and stone location, assessed by a non-contrast computed tomography (CT) scan or ultrasound (US) and kidneyureter-bladder x-ray (KUB).

The analyzed intra-operative parameters were: OT, number of punctures required, intraoperative complications, and exit strategy.

The collected post-operative parameters were: length of hospital stay, post-operative complications (graded by the Clavien-Dindo classification), SFR, and the need for auxiliary procedures [13]. The stone-free status was assessed one month after surgery by means of two radiological exams, ultrasound (US) and kidney, ureter, and bladder (KUB) x-ray, and defined as the abserice of residual fragments $>4 \mathrm{~mm}$ after one month from surgery.

\section{Equipment and procedure}

Before starting the procedure, we administered antibiotic prophylaxis with a third-generation cephalosporin. In case of positive pre-operative urine culture, a argeted antibiotic therapy was scheduled prior to surgery on the basis of the urine culture antibiggram. After general anaesthesia, patients were positioned in Valdivia-Galdakao modified position. The first step was to gain ureteral access. It is our practice to use a 4.8 or 6 Fr open-ended ureteral catheter.

As for the nephrostomy access sheath, we used the clearPetra ${ }^{\circledR}$ system (Well Lead Medical Co., Ltd., China), which is shøwn in Figure 1. This device is available in different measures: internal diameters range from 10 to 22 Fr and lengths can range from 13 to $21 \mathrm{~cm}$. In Milan the 16-Fr x 13$\mathrm{cm}$ access sheath was adopted, while in Barcelona surgeons preferred the $14-\mathrm{Fr} \times 13 \mathrm{~cm}$ system.

A fluoroscopy-guided $(+/$ - ultrasorim $)$ renal puncture was performed by the urologist. The tract was dilated in one-shot yia the CiearPetra $16 \mathrm{Fr}$ access sheath. When the 14 Fr ClearPetra was used a progressive dilation by means of 8-10-12 Fr fascial dilators (Cook, Bloomington, IN, USA) was performed.

We used a 12 Fr nephroscope (Karl Storz SE \& Co. KG, Germany; length: $22 \mathrm{~cm}$; working channel 6.7 Fr) for the 16 Fr ClearPetra, and a 9.5 Fr semirigid ureteroscope (Karl Storz SE \& Co. KG, Germany; length: $34 \mathrm{~cm}$; working channel $5 \mathrm{Fr}$ ) was used for the $14 \mathrm{Fr}$ ClearPetra.

The sheath is equipped with a lateral arm connected to the aspiration pipe which is linked to a $200 \mathrm{ml}$ plastic bottle. The stone collection bottle has a valve that permits the aspiration of the fluids in the aspiration system without losing stone fragments. The aspiration strength can be adjusted in three ways. The first one is through a valve on the connecting tube. The second one is through an oval window on the lateral arm of the sheath that can be completely or partially closed by the connecting 
tube. The third way is closing the oval window on-demand with a finger to temporarily increase the aspiration. The ClearPetra system is therefore characterized by a continuous inflow and a suctioncontrolled outflow.

For estimation purposes, we considered our system as a closed one and estimated the involved variables. In accordance to Bernoulli's principle, the aspiration pressure should be $p=-133 \mathrm{cmH}_{2} \mathrm{O}$ for the 16-Fr nephrostomy sheath/12-Fr nephroscope kit, and $p=-143 \mathrm{cmH}_{2} \mathrm{O}$ for the 14-Fr nephrostomy sheath/9.5-Fr ureteroscope kit (Figure 2; Appendix A). To allow a continuous flow of fluid with controlled intrarenal pressure of $15 \mathrm{cmH}_{2} \mathrm{O}$ as demonstrated by Zanetti et al. [14], the aspiration was set at $\sim 120-150 \mathrm{cmH}_{2} \mathrm{O}$. Lithotripsy was performed with the Holmium:YAG laser (365-550 $\mu \mathrm{m}$ fiber) with a frequency and energy setting of 10-20 Hz and 1-1.5 J, respectively. Litholapaxy was achieved by slowly drawing back the nephroscope inside the sheath uritf the opening of the lateral aspiration arm was reached. A red stripe on the sheath marks the limit for the nephroscope retraction. Baskets or forceps were not routinely used except for stones located distant to the tip of the access sheath and/or located in difficuit-to-reach positions) (e.g. stones located in an upper calyx and renal access through a middiellower calyx). Flexibie nephroscopy was finally performed in case of doubt of residual fragments.

The exit strategy varied according to the specificity of the scenario. A nephrostomy tube was usually left in place and removed after $24-48$ hours. It the procedure was free from complicatons, and no large residual fragment was detected intraoperatively, the nephrostomy tube was not positioned. Instead, an indwelling ureteral catheter was kept in place for 1-2 days and subsequently removed. The bladder catheter was always left in place for at least 24-48 hours.

Analysis of materials costs

All costs for standard materials andmaterial specific of the procedure were analysed. The cost of the ClearPetra® systen was compared to the cost of standard mini-PCNL (MIP set, Karl Storz SE \& Co. KG, Germany), which was amortized on the average number of pediatric PCNL performed in the last two years.

\section{Statistical analysis}

As for the statistical analysis, we estimated the median and interquartile range (IQR) for each quantitative variable, whereas for qualitative variables we reported the occurrence of the various outcomes. Data were stored and analyzed using Microsoft Excel (Microsoft Corporation, Washington, USA). 
Thirteen patients ( 8 males, 5 females) with a median age of 119 months (IQR: $97-160$ ) and a median weight of $29 \mathrm{Kg}$ (IQR: 25-40) were recruited for the study (Table 1). We performed 18 vmPCNLs in 16 renal units - 10 right $(55.6 \%)$ and 8 left (44.4\%). Two patients had a pre-planned two-stage vmPCNL due to stone burden. In one additional case the decision to perform a second-look procedure was made intraoperatively due to the prolongation of the OT. Eight procedures $(44.4 \%)$ were carried out on staghorn stones. Fifteen $(83.3 \%)$ surgeries were performed for multiple rena stones. Six (33.3\%) patients were pre-stented, $2(11.1 \%)$ had a nephrostomy tube prior to the intervention and 2 (11.1\%) had both. The median cumulative stone size was $32 \mathrm{~mm}$ (IQR: 22-46) and was significantly higher in the population from Milan (37 mm vs $19 \mathrm{~mm} ; \mathrm{p}=0.02)$. Seven out thirteen patients $(53.8 \%)$ presented with comorbidities; among them some were predisposed to stone recurrence (e.g. primary hyperoxaluria type 1, cystinuria etc.). One patient had been submitted to a Cohen'sureterovesical reimplantation. No pre-operative blood test revealed anemia or a significant alteration of the renal function. One out of 18 cases presented with a positive pre-pperative urine cuiture and underwent a 7-day-long antibiotic therapy before performing vmPCNI.

\section{Surgical procedure}

The median OT was 128 min (IQR: 99-167; Table 2). In 13 (72.2\%) cases, a single tract dilation was performed; in 4 cases two tracts were necessary, whereas 3 ctracts were only necessary in a single case. A lower-calyx dilation was used in $14(58.3 \%)$ sorgeries, while a first mid-calyx approach was preferred in $7(29.2 \%)$ procedures. A dilation of the upper calyx was performed in $3(12.5 \%)$ vmPCNLs. Stone removal was achieved only viaction in 6/18 (33\%) of cases. At the end of the procedure, a nephrostomy tube was positioned in $83.3 \%(15 / 18)$ of cases. A double-J stent was positioned in $3(16.7 \%)$ cases. All vmPCW Ls were completed without intraoperative complications.

\section{Outcomes}

Fever (5/18; 28\%) was the most frequent post-operative complication. Two (11\%) patients experienced a renal colic after nephrostomy closure or removal. Six (33.3\%) patients presented a minor complícation (Clavien-Dindo $\leq$ II) during post-operative course. One (5.6\%) patient underwent post-operative double-J ureteral stent positioning due to fever and hydronephrosis after catheter removal (Clavien-Dindo IIIa).

The stone composition was as follows: 3 calcium monohydrate oxalate, 3 calcium monohydrate oxalate and calcium carbonate, 2 cystine, and 5 phosphate-based infectious stones.

The median post-operative stay was 3 days (IQR: 3-6 days). No patient required blood transfusions. The SFR was confirmed in 13/16 renal units $(81.3 \%)$. The SFR rised up to $93.8 \%(15 / 16)$ after ancillary procedures, such as second-look vmPCNL $(n=1)$ or RIRS $(n=2)$. The patient affected by 
primary hyperoxaluria presenting with a bilateral urolithiasis, was subjected to one left-sided and two right-sided vmPCNLs. Stone-free status was only obtained in the right kidney.

\section{Analysis of materials costs}

The average cost of a mini-PCNL performed with vmPCNL or mini-PCNL using a reusable set was $734.8 €$ and $710.7 €$, respectively (Table 3$)$. The vmPCNL costed $24.1 €(+3,4 \%)$ more per surgery. The ClearPetra ${ }^{\circledR}$ system costed $256.2 €$ per surgery while standard mini-PCNL set $134.12 €$. The standard materials costed $478.6 €$ for vmPCNL and $576.5 €$ for mini-PCNL using a reusable set, reflecting the less frequent use of the basket in vmPCNLs.

\section{DISCUSSION}

In this study assessing the utility of vmPCNL in the pediatric population affected by complex stones, we demonstrated that this system was safe and feasible in the selected population

Pediatric urolithiasis presents with different challenges from those encountered in the adults. In the pediatric age, stone formation is often associated with anatomical abnormalities, metabolic disorders, and UTIs, all factors that can increase recurrence risk [15,10]. Pediatrie patients can be treated with minimally invasive techniques. However, children with large stme burdens, complete renal staghorn calculi, SWL-refractory stones, dilated or obstructed kidneysare best treated with PCNL.

In order to decrease the morbidity associated with PCN1, over the last few years miniaturized PCNL techniques have gained increased popularity [1]]. The miniaturization of the technique may be particularly beneficial in the pediatric popslation. It has been demonstrated that the renal injury following dilation is significantly wider when the tract is more than 22 French. A tract dilation of 16 French generates a renal fissure of a diaineter of 4.4/6.28 mm in cadaveric/porcine model. On the other hand, a tract dilation of 24 French (standard-PCNL size) generates a renal fissure of 7.49/12.53 $\mathrm{mm}$, almost doubling the diameter of a mini-PCNL and tripling the total dilation area [18]. These findings explain why the tract dilation size is associated with hemorrhagic complications. The impact of a tract dilation on a pediatric kidney, which mean longest diameter is $9 \mathrm{~cm}$ in 10 -year-old patients, is even more significant. However, their superiority in terms of safety and efficacy compared to conventional PCNL is still under debate.

The smaller tract size may be associated with decreased SFRs and, because of poor fluid drainage, elevated intra-renal pressures for a longer OT with respect to standard PCNL, increase the risk of infective complications $[19,20]$.

In children with stones larger than $2 \mathrm{~cm}$, Saad et al. showed that mini-PCNL has a higher SFR than RIRS ( $71 \%$ vs $95.5 \%, \mathrm{p}=0.046)$, but it is nonetheless associated to higher radiation exposure, longer 
hospital stay and more complications [21,22]. The rate of fever in the PCNL group was twice the one of the RIRS group.

To improve the clinical outcomes related to mini-PCNL, research is currently focusing on avoiding high intra-renal pressures [23]. vmPCNL system may control and limit intra-renal pressures above the physiological limit of $30 \mathrm{mmHg}$, beside reducing the dissemination of stones in the pyelocaliceal system during lithotripsy [12,14].

To the best of our knowledge, our study is the first to describe the use of a vmPCNL system in the pediatric population. Our study demonstrated the feasibility of a vmPCNL approach in pediatric patients. No intra-operative complications were recorded. During the post-operative period, no blood transfusions were required, and 5 procedures were complicated by fever, with onser usually happened on the first 24 hours.

Rashid et al. reported an initial SFR after Mini-PCNL for complex staghorn stongs in children of $78 \%$, which increased to $89 \%$ after a few ancillary procedures [24]. Their median OT was 91 min (range 55-130 $\mathrm{min}$ ). Our SFRs (81.3 and 93.8\%) were comparable to the ones reported in the literature. The SFR might be significantly conditioned by patients piedisposing factors to stone formation (Table 1), which were present in $53.8 \%$ ( $7 / 13$ ) of patients enrolled in our study. Moreover, the stone burden was significant (median cumulative stone gize $=32 \mathrm{~mm}$ ). The vmPCNL seems a sustainable procedure as total cost per procedre is slighty higher than a mini-PCNL using a reusable set. Moreover, the average cost of a ymPCI. Resembles the costs reported in literature for percutaneous surgeries, ranging from $562.79 €$ to $749.39 €$ [25]. Our study presents several limitations. To start with, the study design is associated with a low level of evidence, as it is not a comparative study. The sample was relatively small. Both pre-operative stone burden and postoperative staus assessment weres heterogeneous and not performed with the most accurate radiological exams. Indeed, radiological assessment should be performed by CT imaging, as US has a low sensitivity and inability to properly measure stone size [26]. This is in contrast with the pursuit of limiting radiation exposure in children. Which is why we preferred to assess the SFR with US and KUB x-rays limiting radiation exposure. As a result, SFR might have been overestimated. We reported only the largest stone diameter instead of the entire stone volume [27]. The cumulated stone size uses only one dimension, and this may limit its reliability, especially in case of complex stone shape and stones $>20 \mathrm{~mm}[28,29]$.

According to our experience, the continuous aspiration was associated with a clear vision during the procedure. Considering our high-risk population, the limited incidence of infectious complications in our series may be associated with the low intra-renal pressures, warranted by the aspiration system. 
1 Moreover, the facilitated litholapaxy and the absence of fragments scattering, guaranteed by the

2 vmPCNL, may decrease OT and the need for disposable devices.

\section{CONCLUSIONS}

The use of a vmPCNL system seems to be a sustainable, safe and feasible procedure for kidney stones treatment in the pediatric population. Further multi-institutional RCTs are mandatory to test our initial hypothesis and to compare clinical outcomes of mini-PCNL using a reusable set versus vmPCNL.

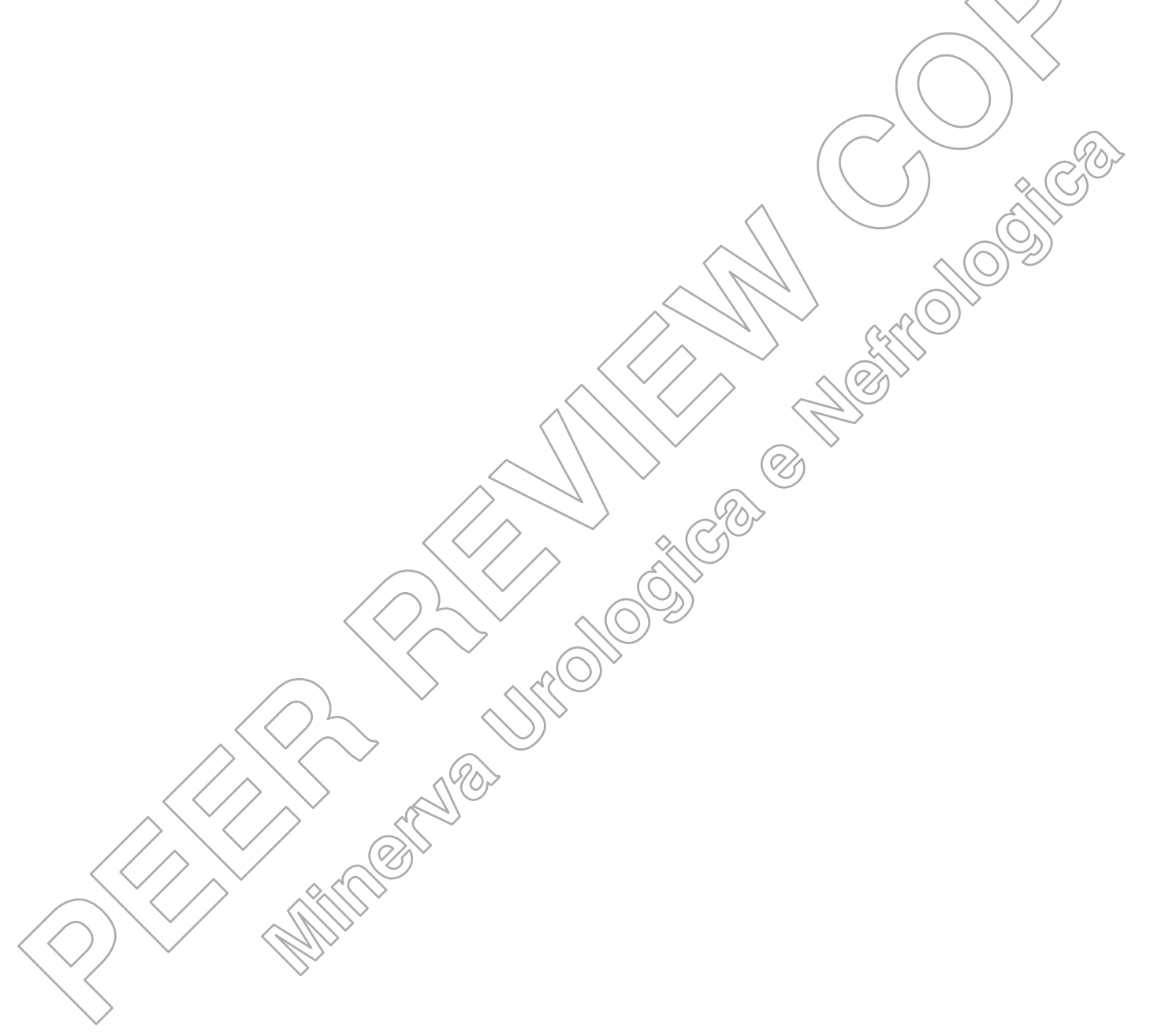


[1] Rassweiler J, Rassweiler MC, Klein J. New technology in ureteroscopy and percutaneous nephrolithotomy. Current Opinion in Urology 2016;26:95-106.

[2] Fernström I, Johansson B. Percutaneous pyelolithotomy. A new extraction technique. Scand J Urol Nephrol 1976;10:257-9.

[3] Sen H, Seckiner I, Bayrak O, Dogan K, Erturhan S. A comparison of micro-PERC and retrograde intrarenal surgery results in pediatric patients with renal stones. Journal of Pediatric Urology 2017;13:619.e1-619.e5.

[4] Ishii H, Griffin S, Somani BK. Flexible ureteroscopy and lasertripsy (FURSL) for paediatric renal calculi: Results from a systematic review. Journal of Pediatric Urology 2014;10:1020-5.

[5] EAU Guidelines. Edn. prese. Arnhem, The Netherlands: EAU Guidelines Office; 2019.

[6] De S, Autorino R, Kim FJ, Zargar H, Laydner H, Balsamo R, et al. Percyataneous

nephrolithotomy versus retrograde intrarenal surgery: A systematic review and meta-analysis. European Urology 2015;67:125-37.

[7] Zanetti SP, Boeri L, Gallioli A, Talso M, Montanari E. Minimally invasive PCNL - MIP. Archivos Espanoles de Urologia 2017;70:226-34.

[8] Li LY, Gao X, Yang M, Li JF, Zhang H Bin, Xu WF, et al. Does a Smaller Tract in Percutaneous Nephrolithotomy Contribute to Less Invasiveness? A Prospective Comparative Study. Urology 2010;75:56-61.

[9] Zhu W, Liu Y, Liu L, Lei M, Yuan J, Wan SP, et al. Minimally invasive versus standard percutaneous nephrolithotomy: a meta-analysis. Urolithiasis 2015;43:563:-70.

[10] Tokas T, Herrmann TRW, Skolarikos A, Nagele T. Pressure matters: intrarenal pressures during normal and pathological conditions, and impact of increased values to renal physiology. World Journal of Urology 2019;37:125-31.

[11] Tokas T, Skolarikos A, Herrmann TRW, Nagele U. Pressive matters 2: intrarenal pressure ranges during upper-tract endourological procedures. World 90 urnal of Urology 2019;37:133-42. [12] Zeng G, Wan S, Zhao Z, Zhu J, Tuerxun A, Song C. et al. Super-mini percutaneous nephrolithotomy (SMP): A new concept in technique and instrumentation. BJU International 2016;117:655-61.

[13] Clavien PA, Barkun J, de Oliveira ML, authey JN, Dindo D, Schulick RD, et al. The Clavien-Dindo Classification of Surgical Complications. Annals of Surgery 2009;250:187-96. [14] Zanetti SP, Lievore E, Fontana M, Turetti M, Gallioli A, Longo F, et al. Vacuum-assisted mini-percutaneous nephrolithotomy: arewy perspective in fragments clearance and intrarenal pressure control. World J Urol 2020.

[15] Bujons A. Millan F, Centenos C, Emiliani E, Sánchez Martín F, Angerri O, et al. Minipercutaneous nephrgil thotomy with high-power holmium YAG laser in pediatric patients with staghorn and complex calculi. Journal of Pediatric Urology 2016;12:253.e1-253.e5.

[16] Berrettini A, Boeri L, Montanari E, Mogiatti M, Acquati P, De Lorenzis E, et al. Retrograde intrarenal surgery using ureteral access sheaths is a safe and effective treatment for renal stones in children weighing $<20 \mathrm{~kg}$. Journal of Pediatric Urology 2018;14:59.e1-59.e6.

[17] Zeng G, Zhu W, Lam W. Miniaturised percutaneous nephrolithotomy: Its role in the treatment of urolithiasis and our experience. Asian Journal of Urology 2018;5:295-302.

[18] Emiliani E, Talso M, Baghdadi M, Traxer O. Renal parenchyma injury after percutaneous nephrolithotomy tract dilatations in pig and cadaveric kidney models. Cent European J Urol 2017;70:69-75. https://doi.org/10.5173/ceju.2017.930.

[19] Kaygisiz O, Satar N, Gürocak S, Kefi A, İzol V, Önal B, et al. Factors predicting postoperative febrile urinary tract infection following percutaneous nephrolithotomy in prepubertal children. Journal of Pediatric Urology 2018;14:448.e1-448.e7.

[20] Loftus CJ, Hinck B, Makovey I, Sivalingam S, Monga M. Mini Versus Standard Percutaneous Nephrolithotomy: The Impact of Sheath Size on Intrarenal Pelvic Pressure and Infectious Complications in a Porcine Model. Journal of Endourology 2018;32:350-3.

[21] Saad KSM, Youssif ME, Hamdy SAIN, Fahmy A, El Din Hanno AG, El-Nahas AR. 
Percutaneous nephrolithotomy vs retrograde intrarenal surgery for large renal stones in pediatric patients: A randomized controlled trial. Journal of Urology 2015;194:1716-20.

[22] Lu P, Song R, Yu Y, Yang J, Qi K, Tao R, et al. Clinical efficacy of percutaneous nephrolithotomy versus retrograde intrarenal surgery for pediatric kidney urolithiasis. Medicine (United States) 2017;96.

[23] Walcher U, Horstmann M, Anastasiadis AG, Nagele U, Hennenlotter J, Sievert K-D, et al. A Newly Designed Amplatz Sheath Decreases Intrapelvic Irrigation Pressure During MiniPercutaneous Nephrolitholapaxy: An in-Vitro Pressure-Measurement and Microscopic Study . Journal of Endourology 2007;21:1113-6.

[24] Rashid AO, Amin SH, Al Kadum MA, Mohammed SK, Buchholz N. Mini-Percutaneous Nephrolithotomy for Complex Staghorn Stones in Children. Urologia Internationalis 2019:1-4. [25] Schoenthaler M, Wilhelm K, Hein S, Adams F, Schlager D, Wetterauer U, et al. Ultra-mini PCNL versus flexible ureteroscopy: a matched analysis of treatment costs (endoscopes and disposables) in patients with renal stones 10-20 mm. World J Urol 2015;33.1601-5.

[26] Ganesan V, De S, Greene D, Torricelli FCM, Monga M. Accuracy of ultrasonography for renal stone detection and size determination: is it good enough for management decisions? BJU International 2017;119:464-9.

[27] De Coninck V, Traxer O. The Time Has Come to Report Stone Burden in Terms of Volume Instead of Largest Diameter. Journal of Endourology 2018:32:265-6.

[28] Ito H, Kawahara T, Terao H, Ogawa T, Yao M, Kubota Y, et al Lutility and limitation of cumulative stone diameter in predicting urinary stone burden at flexible ureteroscopy with holmium laser lithotripsy: a single-center experience. PLoS ONE 2013;8:e65050.

[29] Giusti G, Proietti S, Villa L, Cloutier J, Rosso M, Gadda GM, et al. Current Standard Technique for Modern Flexible Ureteroscopy: Tips and Tricks. Ear Urol 2016;70:188-94.

Conflicts of interest: The Authors declare nothing to disclose.

\section{Authors' contributions:}

Study design: Berrettini A, Bujors A, Manzoni G, Montanari E

Data collection: Galligì A, Sampogna Gu, Quiróz Y, Llorens E

Manuscript writing: Gailioli A, S Ampogna G, Gnech M, De Lorenzis E

Manuscript editing. Bujons A, Montanari E, Palou J, Albo G

Funding: none

Acknowledgements: Carolina Bebi for language revision and Irene Fulgheri for cost analysis. 


\section{TABLES}

Table 1. Baseline characteristics of the population

\begin{tabular}{|c|c|}
\hline Parameters & Values \\
\hline No. patients & $\begin{array}{l}13 \\
-8 \text { Milan }(61.5 \%) \\
-5 \text { Barcelona }(38.5 \%) \\
\end{array}$ \\
\hline No. renal units & 16 \\
\hline No. vmPCNLs & 18 \\
\hline Sex & $\begin{array}{l}\text { - Males: } 8(61.5 \%) \\
\text { - Females: } 5(38.5 \%)\end{array}$ \\
\hline Age (months) median (IQR) & $119(97-160)$ \\
\hline Weight (Kg) median (IQR) & $29(25-40)$ \\
\hline BMI $\left(\mathbf{K g} / \mathbf{m}^{2}\right)$ median (IQR) & $17.7(16.4-18.3)$ \\
\hline Type of stone & $\begin{array}{l}\text { - Staghorn: } 8(44.4 \%) \\
\text { - Multiple: } 15(8.3 \%)\end{array}$ \\
\hline Stone side & $\begin{array}{l}\text { - Right: } 10(55.6 \%) \\
\text { - Left: } 8(44.4 \%)\end{array}$ \\
\hline Cumulative stone size $(\mathbf{m m}) *$ median (IQR) & $32(22-46)$ \\
\hline No. patients with comorbidities & 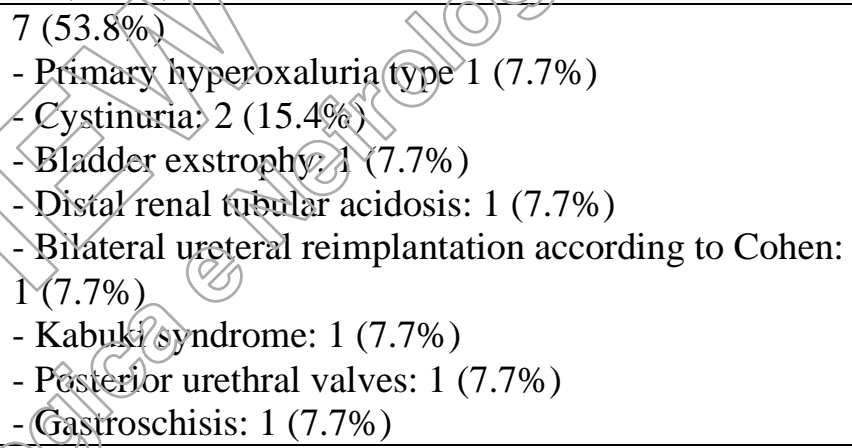 \\
\hline Pre-operative condition & $\begin{array}{l}\text { Oreteral DJ stent: } 6(33.3 \%) \\
\text { - Nephrostomy tube: } 2(11.1 \%) \\
\text { - Ureteral DJ stent + nephrostomy tube: } 2(11.1 \%)\end{array}$ \\
\hline Pre-operative hemogiobin (g/dL) median (IQR) & $13.2(12.1-13.7)$ \\
\hline Pre-operative creatinive (mg/dL) median ( IQR) & $0.7(0.5-1.1)$ \\
\hline Pre-operative sodium (mEq/dL) median (IQR) & $140(138-142)$ \\
\hline Pre-operative potassium (mEq/d] & $4.6(4.3-4.7)$ \\
\hline Pre-operative caicium (mg/dis) médian (IQR) & $9.8(9.6-9.9)$ \\
\hline Pre-openative uric acid (mg/di) median (IQR) & $3.9(3.4-4.2)$ \\
\hline Positive pre-operative urine culture (\%) & $\begin{array}{l}5.6(1 / 18) \\
\text { - Pseudomonas aeruginosa }\end{array}$ \\
\hline
\end{tabular}

Cumulative stone size was evaluated as sum of largest stones diameter $\mathrm{BMI}=$ body mass index; IQR = inter-quartile range; $\mathrm{vmPCNL}=$ semi-closed-circuit vacuumassisted Mini-PCNL. 
Table 2. Intra- and post-operative data of the patients submitted to vmPCNL

\begin{tabular}{|c|c|}
\hline Parameters & Values \\
\hline Operative time (min) median (IQR) & $128(99-167)$ \\
\hline Tract location & $\begin{array}{l}\text { Upper calyx: } 3(12.5) \\
\text { Middle calyx: } 7(29.2) \\
\text { Lower calyx: } 14(58.3)\end{array}$ \\
\hline Tract & $\begin{array}{l}\text { Single: } 13(72.2) \\
\text { Multiple: } 5 \text { (27.8) }\end{array}$ \\
\hline Intra-operative complications (\%) & 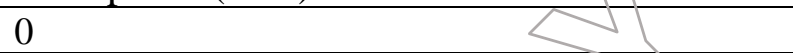 \\
\hline Exit strategy & $\begin{array}{l}\text { - Nephrostomy tube: } 15(83.3 \%) \text { [in one case: two } \\
\text { nephrostomy tubes] } \\
\text { - Tubeless: } 3(16.7 \%) \\
\text { - double-J ureteral stert: } 3(16.7 \%) \\
\text { - single-J ureteral catheter: } 5(27.8 \%)\end{array}$ \\
\hline Stone composition & $\begin{array}{l}\text { - Calcium oxalate monohydrate: } 3(232 \%) \\
\text { - Calcium oxalate monohydrate and cegicium } \\
\text { carbonate } 3(23.1 \%) \\
\text { - Cystipie: } 2(15.4 \%) \\
\text { - Phosphate-based infectious stones (e.g. struvite, } \\
\text { carboapatite): } 5(38.5 \%)(0)\end{array}$ \\
\hline Fever $(\%)$ & $27.8(5 / 18)$ \\
\hline Blood transfusion $(\%)$ & $0>2$ \\
\hline $\begin{array}{l}\text { Complications according to Clavien-Dindo } \\
\text { classification }(\%) \\
\text { - Grade I-II } \\
\text { - Grade IIIa }\end{array}$ & $\begin{array}{l}-33.3(6,18) \\
-56.6(1 / 18)\end{array}$ \\
\hline Hospital stay (days) median (IQR) & $3(3-6)$ \\
\hline Post-operative hemoglobin (g/dL) median (I $\mathrm{R} \mathrm{R})^{*}$ & (2). \\
\hline Post-operative creatinine (mg/dL) median (IQR)** & $0.61(0.52-0.86)$ \\
\hline Post-operative CRP $(\mathbf{m g} / \mathbf{L} /)$ median $(\mathrm{IQR}) * *$ * & $3.3(1.8-4.8)$ \\
\hline Stone-free rate $(\%)$ & $81.3(13 / 16)$ \\
\hline Stone-free rate after anciliary procedures $(\%)$ & $93.8(15 / 16)$ \\
\hline
\end{tabular}

vmPCNL = semi-ciosed-circuit vacuum-assisted Mini-PCNL; CRP = C-reactive protein; IQR = interquartile range 
Table 3. Analysis of the disposable and reusable materials cost for mini-PCNL, stratified for miniPCNL using a reusable set (standard) and vacuum-assisted mini-PCNL (excluding endoscopes)

\begin{tabular}{|c|c|c|c|c|}
\hline Materials & Quantity & Price $(€)$ & $\begin{array}{c}\text { Standard } \\
\text { mini-PCNL }(€)\end{array}$ & $\begin{array}{c}\text { Vacuum-assisted } \\
\text { mini-PCNL }(€)\end{array}$ \\
\hline Ureteral catheter & 1 & 10.0 & 10.0 & 10.0 \\
\hline Hydrophilic wire & 2 & 24.3 & 48.6 & 48.6 \\
\hline Basket & 1 & 146.2 & 146.2 & 48.3 \\
\hline Laser fiber* & 1 & 888.2 & 88.8 & 88.8 \\
\hline Nephrostomy set & 1 & 73.2 & 73.2 & 73.2 \\
\hline Irrigation set** & 1 & 136.1 & 78.4 & 78.4 \\
\hline Contrast & 1 & 29.3 & 29.3 & 29.3 \\
\hline Lubrication & 2 & 1.9 & 3.8 & 3.8 \\
\hline Gowns/gloves & 5 & 2.5 & 12.7 & 12.7 \\
\hline Draping & 1 & 62 & $62.0(0$ & 62.0 \\
\hline Miscellaneous & I & 23 & 23.6 & 23.6 \\
\hline PCNL set*** & 1 & & 134.1 & l \\
\hline ClearPetra & 1 & 256.2 & I & 256.2 \\
\hline Total & 12 & 1 & 710.7 & 734.8 \\
\hline
\end{tabular}

* Depreciation calculated on 10 procedures

** Depreciation calculated on 4 procedures

***Depreciation calculated on the number of pediatric PCNLs performed in the last 24 months 


\section{TITLES OF FIGURES}

Figure 1. A) The ClearPetra® system (Well Lead Medical Co., Ltd., China), is shown. A plug is put over the external access to prevent the medium from flowing out. The sheath is equipped with a lateral arm connected to the aspiration system through a $200 \mathrm{ml}$ plastic bottle, which collects stone fragments. B) The pressure vent on the lateral arm is used to regulate aspiration and the red stripe on the sheath is the mark for endoscope retraction. D) Lapaxy is performed by slowly drawing back the nephroscope inside the sheath until the red stripe on the sheath, thus aspiring the fragments in the lateral arm.

Figure 2. Estimation of the pressures involved in the empioyed system. The entire circuit was considered as closed for estimation purposes. Bernoulii's principle states the total pressure is a constant in the system and derives from the sum of static and dynamic pressures. In the example shown, we considered the 16-Fr nephrostomy sheath and 12-Fr nephroseope, evaluating the aspiration pressure in $p=-13.013 \mathrm{~Pa}=-133 \mathrm{cmH}_{2} \mathrm{O}$. To estimate the presme with a 9.5-Fr ureteroscope and a 14-Fr nephrostomy sheath, the values of $r_{1}$ and $r_{2}$ were forodified accordingly, obtaining a final aspiration pressure $p=-14.00 \mathrm{~Pa}=-143 \mathrm{CmH}_{2} \mathrm{O}$. 


\section{Semi-closed-circuit vacuum-assisted mini percutaneous nephrolithotomy in the pediatric} population: the initial experience of two tertiary referral centers

Andrea Gallioli ${ }^{*}$, Alfredo Berrettini ${ }^{2}$, Gianluca Sampogna ${ }^{1,2}$, Erika Llorens ${ }^{3}$, Yesica Quiróz ${ }^{3}$, Michele Gnech ${ }^{2}$, Elisa De Lorenzis ${ }^{5}$, Giancarlo Albo ${ }^{5}$, Joan Palou ${ }^{4}$, Gianantonio Manzoni², Anna Bujons $^{3}$, Emanuele Montanari ${ }^{5}$

${ }^{1}$ Urology Unit, Fondazione IRCCS Ca’ Granda Ospedale Maggiore Policlinice, University of Milan, Milan, Italy

${ }^{2}$ Pediatric Urology Unit, Fondazione IRCCS Ca' Granda Ospedale Maggiore Policliniceo, Milan, Italy

${ }^{3}$ Pediatric Urology Division, Fundaciò Puigvert, Barcelona, Spain

${ }^{4}$ Urology Department, Fundació Puigvert, Barcelona, Spain

${ }^{5}$ Urology Unit, Fondazione IRCCS Ca' Granda Ospedale Maggiore Policlinico, Department of Clinical Sciences and Community Health, University of Milan, Digilan, Italy

*Corresponding author: Andrea Gallioli, MD

Urology Unit, Fondazione IRCES Ca' Granda Ospedale Maggiore Policlinico.

Via della Commenda 15.

Milan 20122, Italy.

Tel. +393496645072 .

E-mail address: andrea.gallioli@ginail.com

Keywords: percutaneous nephrolithotomy; urolithiasis; litholapaxy; children; kidney calculi 


\section{ABSTRACT}

\section{Background}

Percutaneous nephrolithotomy (PCNL) is the gold-standard for complex renal stones treatment in the pediatric population. While the miniaturization of PCNL reduces the risk of bleeding, it can hinder surgical and functional outcomes. The aim of the study is to assess the safety and feasibility of semiclosed-circuit vacuum-assisted Mini-PCNL (vmPCNL) in pediatric patients.

\section{Methods}

From January 2017 to December 2018, we prospectively collected data on consecutive vmPCNLs from two European tertiary referral centers. The procedure was performed with the $e$ learPetra ${ }^{\circledR}$ access sheath equipped with a lateral arm connected to the aspiration system (pressure setting 120$150 \mathrm{cmH}_{2} \mathrm{O}$ ) by a $200 \mathrm{ml}$ plastic stone collector. Pre-, intra- and post-operative data and costs were analyzed. The stone-free rate (SFR) was defined as absence of residual fragments $>4 \mathrm{~mm}$ with either ultrasound or kidney, ureter, and bladder x-ray.

\section{Results}

Eighteen vmPCNLs were performed in 16 renal units of 13 children. The median age was 119 months (IQR: 97-160) and the weight was $20 \mathrm{Kg}$ (IQR: 25040$)$. The median cumulative stone size was 32 mm (22-46) with 8 (44.4\%) cases of staghorn stones. The OT was 128 min (IQR: 99-167). The basketing was unnecessary in 6/18 (33\%) cases. Neither intra-operative complications nor blood transfusions occurred. Pgst-operative fever was observed in 5/18 (27.8\%) cases; in one case a double J ureteral stent was placed for coneomitant hydronephrosis. The SFR was $81.3 \%(13 / 16)$, rising to 93.8\% (15/15) after anciliary procedures. The materials costs of a vmPCNL (734.8 €) were comparable to mini-PCNL using a reusable set $(710.7 €)$.

\section{Conclusions}

The vmPCNL seems to be sustainable, safe and feasible for kidney stones treatment in the pediatric population. 


\section{INTRODUCTION}

Surgical treatment of pediatric kidney stones has changed over the years [1]. Percutaneous nephrolithotomy (PCNL) was firstly introduced in 1976 and it still has a leading role despite many technological advancements in retrograde approaches [2-4]. According to the EAU guidelines, PCNL remains the first surgical option for renal stones $>20 \mathrm{~mm}\left(\sim 300 \mathrm{~mm}^{2}\right)$, for lower pole cáliceal stones $>10 \mathrm{~mm}$ and for staghorn or multiple stones [5]. It ensures a higher stone-free rate (SFR) than shockwave lithotripsy (SWL) and retrograde intra-renal surgery (RIRS), despice presenting a higher rate of major complications and a higher decrease of post-operative hemogløbin levels [6]. In order to reduce PCNL morbidity, Jackman et al. introduced the Mini-PCNL in 1998, reporting encouraging results in terms of complication rate reduction. Their findings were subsequently confirmed by several randomized controlled trials (RCTs) [7. Although mini-PCDIt (nephrostomy tract size $<20 \mathrm{Fr}$ ) may reduce the number of major complications (haemorrhage in particular), some authors argue that it may decrease the SFR, lengthen the operative time (OT, and increase the number of post-operative urinary tract infections (UTI) [8,9].

Endourological research is currently focusing on regulating intra-renal pressures, especially, to reduce the risk of infection. During surgery, irrigation flgoy and irrigation pressures tend to be increased in order to achieve better visibility/10]. Increased intrarenal pressures can provoke the deterioration of the renal parenchyma, and causepyelorenal backflow, fluid reabsorption, and bacteremia [11]. Novel systems to control and timit the increase of intra-renal pressures, like a semiclosed-circuit vacuum-assisted mini-PCNL (mPCNL) system, may potentially reduce the number of post-operative compications and bvercome some of the above-mentioned limitations of a minimally in asive approaches [12.

The aim of the study is to report our initial experience using a vmPCNL system for renal stone treatment and to assess its safety and efficacy in pediatric patients.

\section{MATERIALS AND METHODS}

We prospectively collected data from two European tertiary referral centers: Fondazione IRCCS Ca' Granda - Ospedale Maggiore Policlinico (Milan) and Fundació Puigvert (Barcelona). From January 2017 to December 2018, we enrolled to the study all consecutive < 18 years old at the time of surgery undergoing a vmPCNL. Patients' data and surgical outcomes were retrospectively analyzed. The study was conducted in accordance with the Declaration of Helsinki (1964) and its later amendments. The study was approved by the Institutional Review Board. All patients signed an informed consent at the time of hospitalization to share anonymously clinical information for research purposes. 


\section{Data collection}

Pre-operative patient data included demographic variables (e.g. age, sex), medical history and stone characteristics, like cumulative stone size (evaluated as sum of largest stones diameter) and stone location, assessed by a non-contrast computed tomography (CT) scan or ultrasound (US) and kidneyureter-bladder x-ray (KUB).

The analyzed intra-operative parameters were: OT, number of punctures required, intraoperative complications, and exit strategy.

The collected post-operative parameters were: length of hospital stay, post-operative complications (graded by the Clavien-Dindo classification), SFR, and the need for auxiliary procedures [13]. The stone-free status was assessed one month after surgery by means of two radiological exams, ultrasound (US) and kidney, ureter, and bladder (KUB) x-ray, and defined as the abserice of residual fragments $>4 \mathrm{~mm}$ after one month from surgery.

\section{Equipment and procedure}

Before starting the procedure, we administered antibiotic prophylaxis with a third-generation cephalosporin. In case of positive pre-operative urine culture, a rargeted antibiotic therapy was scheduled prior to surgery on the basis of the urine culture antibiggram. After general anaesthesia, patients were positioned in Valdivia-Galdakao modified position. The first step was to gain ureteral access. It is our practice to use a 4.8 or 6 Fr open-ended ureteral catheter.

As for the nephrostomy access sheath, we used the clearPetra ${ }^{\circledR}$ system (Well Lead Medical Co., Ltd., China), which is shøwn in Figure 1. This device is available in different measures: internal diameters range from 10 to 22 Fry and lengths can range from 13 to $21 \mathrm{~cm}$. In Milan the 16-Fr x 13$\mathrm{cm}$ access sheath was adopted, while in Barcelona surgeons preferred the $14-\mathrm{Fr} \times 13 \mathrm{~cm}$ system.

A fluoroscopy-guided $(+/$ - ultrasorim $)$ renal puncture was performed by the urologist. The tract was dilated in one-shot yia the CiearPetra $16 \mathrm{Fr}$ access sheath. When the 14 Fr ClearPetra was used a progressive dilation by means of 8-10-12 Fr fascial dilators (Cook, Bloomington, IN, USA) was performed.

We used a 12 Fr nephroscope (Karl Storz SE \& Co. KG, Germany; length: $22 \mathrm{~cm}$; working channel 6.7 Fr) for the 16 Fr ClearPetra, and a 9.5 Fr semirigid ureteroscope (Karl Storz SE \& Co. KG, Germany; length: $34 \mathrm{~cm}$; working channel $5 \mathrm{Fr}$ ) was used for the $14 \mathrm{Fr}$ ClearPetra.

The sheath is equipped with a lateral arm connected to the aspiration pipe which is linked to a $200 \mathrm{ml}$ plastic bottle. The stone collection bottle has a valve that permits the aspiration of the fluids in the aspiration system without losing stone fragments. The aspiration strength can be adjusted in three ways. The first one is through a valve on the connecting tube. The second one is through an oval window on the lateral arm of the sheath that can be completely or partially closed by the connecting 
tube. The third way is closing the oval window on-demand with a finger to temporarily increase the aspiration. The ClearPetra system is therefore characterized by a continuous inflow and a suctioncontrolled outflow.

For estimation purposes, we considered our system as a closed one and estimated the involved variables. In accordance to Bernoulli's principle, the aspiration pressure should be $p=-133 \mathrm{cmH}_{2} \mathrm{O}$ for the 16-Fr nephrostomy sheath/12-Fr nephroscope kit, and $p=-143 \mathrm{cmH}_{2} \mathrm{O}$ for the 14-Fr nephrostomy sheath/9.5-Fr ureteroscope kit (Figure 2; Appendix A). To allow a continuous flow of fluid with controlled intrarenal pressure of $15 \mathrm{cmH}_{2} \mathrm{O}$ as demonstrated by Zanetti et al. [14], the aspiration was set at $\sim 120-150 \mathrm{cmH}_{2} \mathrm{O}$. Lithotripsy was performed with the Holmium: YAG laser (365-550 $\mu \mathrm{m}$ fiber) with a frequency and energy setting of 10-20 Hz and 1-1.5 J, respectively. Litholapaxy was achieved by slowly drawing back the nephroscope inside the sheath uritf the opening of the lateral aspiration arm was reached. A red stripe on the sheath marks the limit for the nephroscope retraction. Baskets or forceps were not routinely used except for stones located distant to the tip of the access sheath and/or located in difficult-to-reach positions) (e.g. stones located in an upper calyx and renal access through a middiellower calyx). Flexibie nephroscopy was finally performed in case of doubt of residual fragments.

The exit strategy varied according to the specificity of the scenario. A nephrostomy tube was usually left in place and removed after $24-48$ hours. It the procedure was free from complicatons, and no large residual fragment was detected intraoperatively, the nephrostomy tube was not positioned. Instead, an indwelling ureteral catheter was kept in place for 1-2 days and subsequently removed. The bladder catheter was alyays left in place for at least 24-48 hours.

Analysis of materials eosts

All costs for standard materials andmaterial specific of the procedure were analysed. The cost of the ClearPetraß systen was compared to the cost of standard mini-PCNL (MIP set, Karl Storz SE \& Co. KG, Germany), which was amortized on the average number of pediatric PCNL performed in the last two years.

\section{Statistical analysis}

As for the statistical analysis, we estimated the median and interquartile range (IQR) for each quantitative variable, whereas for qualitative variables we reported the occurrence of the various outcomes. Data were stored and analyzed using Microsoft Excel (Microsoft Corporation, Washington, USA). 
Thirteen patients ( 8 males, 5 females) with a median age of 119 months (IQR: $97-160$ ) and a median weight of $29 \mathrm{Kg}$ (IQR: 25-40) were recruited for the study (Table 1). We performed 18 vmPCNLs in 16 renal units - 10 right $(55.6 \%)$ and 8 left (44.4\%). Two patients had a pre-planned two-stage vmPCNL due to stone burden. In one additional case the decision to perform a second-look procedure was made intraoperatively due to the prolongation of the OT. Eight procedures $(44.4 \%)$ were carried out on staghorn stones. Fifteen $(83.3 \%)$ surgeries were performed for multiple rena stones. Six (33.3\%) patients were pre-stented, $2(11.1 \%)$ had a nephrostomy tube prior to the intervention and 2 (11.1\%) had both. The median cumulative stone size was $32 \mathrm{~mm}$ (IQR: 22-46) and was significantly higher in the population from Milan (37 mm vs $19 \mathrm{~mm} ; \mathrm{p}=0.02)$. Seven out thirteen patients $(53.8 \%)$ presented with comorbidities; among them some were predisposed to stone recurrence (e.g. primary hyperoxaluria type 1, cystinuria etc.). One patient had been submitted to a Cohen'sureterovesical reimplantation. No pre-operative blood test revealed anemia or a significant alteration of the renal function. One out of 18 cases presented with a positive pre-pperative urine cutture and underwent a 7-day-long antibiotic therapy before performing vmPCNI.

\section{Surgical procedure}

The median OT was 128 min (IQR: 99-167; Table 2). In 13 (72.2\%) cases, a single tract dilation was performed; in 4 cases two tracts were necessary, whereas 3 ctracts were only necessary in a single case. A lower-calyx dilation was used in $14(58.3 \%)$ sorgeries, while a first mid-calyx approach was preferred in $7(29.2 \%)$ procedures. A dilation of the upper calyx was performed in $3(12.5 \%)$ vmPCNLs. Stone removal was achieved only viaction in 6/18 (33\%) of cases. At the end of the procedure, a nephrostomy tube was positioned in $83.3 \%(15 / 18)$ of cases. A double-J stent was positioned in $3(16.7 \%)$ cases. All vmPCW Ls were completed without intraoperative complications.

\section{Outcomes}

Fever (5/18; 28\%) was the most frequent post-operative complication. Two (11\%) patients experienced a renal colic after nephrostomy closure or removal. Six (33.3\%) patients presented a minor complícation (Clavien-Dindo $\leq$ II) during post-operative course. One (5.6\%) patient underwent post-operative double-J ureteral stent positioning due to fever and hydronephrosis after catheter removal (Clavien-Dindo IIIa).

The stone composition was as follows: 3 calcium monohydrate oxalate, 3 calcium monohydrate oxalate and calcium carbonate, 2 cystine, and 5 phosphate-based infectious stones.

The median post-operative stay was 3 days (IQR: 3-6 days). No patient required blood transfusions. The SFR was confirmed in 13/16 renal units $(81.3 \%)$. The SFR rised up to $93.8 \%(15 / 16)$ after ancillary procedures, such as second-look vmPCNL $(n=1)$ or RIRS $(n=2)$. The patient affected by 
primary hyperoxaluria presenting with a bilateral urolithiasis, was subjected to one left-sided and two right-sided vmPCNLs. Stone-free status was only obtained in the right kidney. Analysis of materials costs

The average cost of a mini-PCNL performed with vmPCNL or mini-PCNL using a reusable set was $734.8 €$ and $710.7 €$, respectively (Table 3$)$. The vmPCNL costed $24.1 €(+3,4 \%)$ more per surgery. The ClearPetra ${ }^{\circledR}$ system costed $256.2 €$ per surgery while standard mini-PCNL set $134.12 €$. The standard materials costed $478.6 €$ for vmPCNL and $576.5 €$ for mini-PCNL using a reusable set, reflecting the less frequent use of the basket in vmPCNLs.

\section{DISCUSSION}

In this study assessing the utility of vmPCNL in the pediatric population affected by complex stones, we demonstrated that this system was safe and feasible in the selected population

Pediatric urolithiasis presents with different challenges from those encountered in the adults. In the pediatric age, stone formation is often associated with anatomical abnormalities, metabolic disorders, and UTIs, all factors that can increase recurrence risk [15,15]. Pediatrie patients can be treated with minimally invasive techniques. However, children with large stme burdens, complete renal staghorn calculi, SWL-refractory stones, dilated or obstructed kidneysare best treated with PCNL.

In order to decrease the morbidity associated with PCN1, over the last few years miniaturized PCNL techniques have gained increased popularity [1]]. The miniaturization of the technique may be particularly beneficial in the pediatrie popalation. It has been demonstrated that the renal injury following dilation is signiricantly wider when the tract is more than 22 French. A tract dilation of 16 French generates a renal fissure of a diantheter of 4.4/6.28 mm in cadaveric/porcine model. On the other hand, a tract-dilation of 24 Fipench (standard-PCNL size) generates a renal fissure of 7.49/12.53 $\mathrm{mm}$, almost doubling the diameter of a mini-PCNL and tripling the total dilation area [18]. These findings explain why the tract dilation size is associated with hemorrhagic complications. The impact of a tract dilation on a pediatric kidney, which mean longest diameter is $9 \mathrm{~cm}$ in 10 -year-old patients, is even more significant. However, their superiority in terms of safety and efficacy compared to conventional PCNL is still under debate.

The smaller tract size may be associated with decreased SFRs and, because of poor fluid drainage, elevated intra-renal pressures for a longer OT with respect to standard PCNL, increase the risk of infective complications [19,20].

In children with stones larger than $2 \mathrm{~cm}$, Saad et al. showed that mini-PCNL has a higher SFR than RIRS ( $71 \%$ vs $95.5 \%, \mathrm{p}=0.046)$, but it is nonetheless associated to higher radiation exposure, longer 
hospital stay and more complications [21,22]. The rate of fever in the PCNL group was twice the one of the RIRS group.

To improve the clinical outcomes related to mini-PCNL, research is currently focusing on avoiding high intra-renal pressures [23]. vmPCNL system may control and limit intra-renal pressures above the physiological limit of $30 \mathrm{mmHg}$, beside reducing the dissemination of stones in the pyelocaliceal system during lithotripsy [12,14].

To the best of our knowledge, our study is the first to describe the use of a vmPCNL system in the pediatric population. Our study demonstrated the feasibility of a vmPCNL approach in pediatric patients. No intra-operative complications were recorded. During the post-operative period, no blood transfusions were required, and 5 procedures were complicated by fever, with onser usually happened on the first 24 hours.

Rashid et al. reported an initial SFR after Mini-PCNL for complex staghorn stonges in children of $78 \%$, which increased to $89 \%$ after a few ancillary procedures [24]. Their median OT was 91 min (range 55-130 $\mathrm{min}$ ). Our SFRs (81.3 and 93.8\%) were comparable to the ones reported in the literature. The SFR might be significantly conditioned by patients piedisposing factors to stone formation (Table 1), which were present in $53.8 \%$ ( $7 / 13$ ) of patients enrolled in our study. Moreover, the stone burden was significant (median cumulative stone size $=32 \mathrm{~mm}$ ). The vmPCNL seems a sustainable procedure as total cost perprocedure is slighty higher than a mini-PCNL using a reusable set. Moreover, the average cost of a ymPCNI resembles the costs reported in literature for percutaneous surgeries, ranging from $562.79 €$ to $749.39 €$ [25]. Our study presents several limitations. To start with, the study design is associated with a low level of evidence, as it is not a comparative study. The sample was relatively small. Both pre-operative stone burden and postoperative staus assessment weres heterogeneous and not performed with the most accurate radiological exams. Indeed, madiological assessment should be performed by CT imaging, as US has a low sensitivity and inability to properly measure stone size [26]. This is in contrast with the pursuit of limiting radiation exposure in children. Which is why we preferred to assess the SFR with US and KUB x-rays limiting radiation exposure. As a result, SFR might have been overestimated. We reported only the largest stone diameter instead of the entire stone volume [27]. The cumulated stone size uses only one dimension, and this may limit its reliability, especially in case of complex stone shape and stones $>20 \mathrm{~mm}[28,29]$.

According to our experience, the continuous aspiration was associated with a clear vision during the procedure. Considering our high-risk population, the limited incidence of infectious complications in our series may be associated with the low intra-renal pressures, warranted by the aspiration system. 
1 Moreover, the facilitated litholapaxy and the absence of fragments scattering, guaranteed by the vmPCNL, may decrease OT and the need for disposable devices.

\section{CONCLUSIONS}

The use of a vmPCNL system seems to be a sustainable, safe and feasible procedure for kidney stones treatment in the pediatric population. Further multi-institutional RCTs are mandatory to test our initial hypothesis and to compare clinical outcomes of mini-PCNL using a reusable set versus vmPCNL.

\section{REFERENCES}


[1] Rassweiler J, Rassweiler MC, Klein J. New technology in ureteroscopy and percutaneous nephrolithotomy. Current Opinion in Urology 2016;26:95-106.

[2] Fernström I, Johansson B. Percutaneous pyelolithotomy. A new extraction technique. Scand J Urol Nephrol 1976;10:257-9.

[3] Sen H, Seckiner I, Bayrak O, Dogan K, Erturhan S. A comparison of micro-PERC and retrograde intrarenal surgery results in pediatric patients with renal stones. Journal of Pediatric Urology 2017;13:619.e1-619.e5.

[4] Ishii H, Griffin S, Somani BK. Flexible ureteroscopy and lasertripsy (FURSL) for paediatric renal calculi: Results from a systematic review. Journal of Pediatric Urology 2014;10:1020-5.

[5] EAU Guidelines. Edn. prese. Arnhem, The Netherlands: EAU Guidelines Office; 2019.

[6] De S, Autorino R, Kim FJ, Zargar H, Laydner H, Balsamo R, et al. Percutaneous

nephrolithotomy versus retrograde intrarenal surgery: A systematic review and meta-analysis. European Urology 2015;67:125-37.

[7] Zanetti SP, Boeri L, Gallioli A, Talso M, Montanari E. Minimally invasive PCNL - MIP. Archivos Espanoles de Urologia 2017;70:226-34.

[8] Li LY, Gao X, Yang M, Li JF, Zhang H Bin, Xu WF, et al. Does a Smaller Tract in Percutaneous Nephrolithotomy Contribute to Less Invasiveness? A Prospective Comparative Study. Urology 2010;75:56-61.

[9] Zhu W, Liu Y, Liu L, Lei M, Yuan J, Wan SP, et al. Minimally invasive versus standard percutaneous nephrolithotomy: a meta-analysis. Urolithiasis 2015;43:563:-70.

[10] Tokas T, Herrmann TRW, Skolarikos A, Nagele T. Pressure matters: intrarenal pressures during normal and pathological conditions, and impact of increased values to renal physiology. World Journal of Urology 2019;37:125-31.

[11] Tokas T, Skolarikos A, Herrmann TRW, Nagele U. Pressive matters 2: intrarenal pressure ranges during upper-tract endourological procedures. World \$ournal of Urology 2019;37:133-42. [12] Zeng G, Wan S, Zhao Z, Zhu J, Tuerxun A, Song C. et al. Super-mini percutaneous nephrolithotomy (SMP): A new concept in technique and instrumentation. BJU International 2016;117:655-61.

[13] Clavien PA, Barkun J, de Oliveira ML, authey JN, Dindo D, Schulick RD, et al. The Clavien-Dindo Classification of Surgical Complications. Annals of Surgery 2009;250:187-96. [14] Zanetti SP, Lievore E, Fontana M, Turetti M, Gallioli A, Longo F, et al. Vacuum-assisted mini-percutaneous nephrohithotomy: areny perspective in fragments clearance and intrarenal pressure control. World J Urol 2020.

[15] Bujons A. Millan F, Centenos C, Emiliani E, Sánchez Martín F, Angerri O, et al. Minipercutaneous nephrgil thotomy with high-power holmium YAG laser in pediatric patients with staghorn and complex calculi. Journal of Pediatric Urology 2016;12:253.e1-253.e5.

[16] Berrettini A, Boeri L, Montanari E, Mogiatti M, Acquati P, De Lorenzis E, et al. Retrograde intrarenal surgery using ureteral access sheaths is a safe and effective treatment for renal stones in children weighing $<20 \mathrm{~kg}$. Journal of Pediatric Urology 2018;14:59.e1-59.e6.

[17] Zeng G, Zhu W, Lam W. Miniaturised percutaneous nephrolithotomy: Its role in the treatment of urolithiasis and our experience. Asian Journal of Urology 2018;5:295-302.

[18] Emiliani E, Talso M, Baghdadi M, Traxer O. Renal parenchyma injury after percutaneous nephrolithotomy tract dilatations in pig and cadaveric kidney models. Cent European J Urol 2017;70:69-75. https://doi.org/10.5173/ceju.2017.930.

[19] Kaygisız O, Satar N, Gürocak S, Kefi A, İzol V, Önal B, et al. Factors predicting postoperative febrile urinary tract infection following percutaneous nephrolithotomy in prepubertal children. Journal of Pediatric Urology 2018;14:448.e1-448.e7.

[20] Loftus CJ, Hinck B, Makovey I, Sivalingam S, Monga M. Mini Versus Standard Percutaneous Nephrolithotomy: The Impact of Sheath Size on Intrarenal Pelvic Pressure and Infectious Complications in a Porcine Model. Journal of Endourology 2018;32:350-3.

[21] Saad KSM, Youssif ME, Hamdy SAIN, Fahmy A, El Din Hanno AG, El-Nahas AR. 
Percutaneous nephrolithotomy vs retrograde intrarenal surgery for large renal stones in pediatric patients: A randomized controlled trial. Journal of Urology 2015;194:1716-20.

[22] Lu P, Song R, Yu Y, Yang J, Qi K, Tao R, et al. Clinical efficacy of percutaneous nephrolithotomy versus retrograde intrarenal surgery for pediatric kidney urolithiasis. Medicine (United States) 2017;96.

[23] Walcher U, Horstmann M, Anastasiadis AG, Nagele U, Hennenlotter J, Sievert K-D, et al. A Newly Designed Amplatz Sheath Decreases Intrapelvic Irrigation Pressure During MiniPercutaneous Nephrolitholapaxy: An in-Vitro Pressure-Measurement and Microscopic Study . Journal of Endourology 2007;21:1113-6.

[24] Rashid AO, Amin SH, Al Kadum MA, Mohammed SK, Buchholz N. Mini-Percutaneous Nephrolithotomy for Complex Staghorn Stones in Children. Urologia Internationalis 2019:1-4. [25] Schoenthaler M, Wilhelm K, Hein S, Adams F, Schlager D, Wetterauer U, et al. Ultra-mini PCNL versus flexible ureteroscopy: a matched analysis of treatment costs (endoscopes and disposables) in patients with renal stones 10-20 mm. World J Urol 2015;33.1601-5.

[26] Ganesan V, De S, Greene D, Torricelli FCM, Monga M. Accuraey of ultrasonography for renal stone detection and size determination: is it good enough for management decisions? BJU International 2017;119:464-9.

[27] De Coninck V, Traxer O. The Time Has Come to Report Stone Burden in Terms of Volume Instead of Largest Diameter. Journal of Endourology 2018:32:265-6.

[28] Ito H, Kawahara T, Terao H, Ogawa T, Yao M, Kubota Y, et al Utitity and limitation of cumulative stone diameter in predicting urinary stone burden at flexible ureteroscopy with holmium laser lithotripsy: a single-center experience. PLoS ONE 2013;8:e65050.

[29] Giusti G, Proietti S, Villa L, Cloutier J, Rosso M, Gadda GM, et al. Current Standard Technique for Modern Flexible Ureteroscopy: Tips and Tricks. Ear Urol 2016;70:188-94.

Conflicts of interest: The Authors declare nothing to disclose.

\section{Authors' contributions:}

Study design: Berrettini A, Bujors A, Manzoni G, Montanari E

Data collection: Galligì A, Sampogna Gu, Quiróz Y, Llorens E

Manuscript writing: Gailioli A, S Ampogna G, Gnech M, De Lorenzis E

Manuscript editing. Bujons A. Montanari E, Palou J, Albo G

Funding: none

Acknowledgements: Carolina Bebi for language revision and Irene Fulgheri for cost analysis. 
Table 1. Baseline characteristics of the population

\begin{tabular}{|c|c|}
\hline Parameters & Values \\
\hline No. patients & $\begin{array}{l}13 \\
-8 \text { Milan }(61.5 \%) \\
-5 \text { Barcelona }(38.5 \%)\end{array}$ \\
\hline No. renal units & 16 \\
\hline No. vmPCNLs & 18 \\
\hline Sex & $\begin{array}{l}\text { - Males: } 8(61.5 \%) \\
\text { - Females: } 5(38.5 \%)\end{array}$ \\
\hline Age (months) median (IQR) & $119(97-160)$ \\
\hline Weight (Kg) median (IQR) & $29(25-40)$ \\
\hline BMI $\left(\mathbf{K g} / \mathbf{m}^{2}\right)$ median (IQR) & $17.7(16.4-18.3)$ \\
\hline Type of stone & $\begin{array}{l}\text { - Staghorn: } 8(44.4 \%) \\
\text { - Multiple: } 15(83.3 \%)\end{array}$ \\
\hline Stone side & $\begin{array}{l}\text { - Right: } 10(55.6 \%) \\
\text { - Left: } 8(44.4 \%)\end{array}$ \\
\hline Cumulative stone size $(\mathbf{m m}) *$ median $(\mathrm{IQR})$ & $32(22-46)$ \\
\hline No. patients with comorbidities & $\begin{array}{l}7(53.8 \%) \\
\text { - Primary nyperoxaluria type } 1(7.7 \%) \\
\text { - Cystinuria: } 2(15.4 \%) \\
\text { - Bladder exstrophy } 17.7 \%) \\
\text { - Distal renal tubutar acidosis: } 1(7.7 \%) \\
\text { - Bilateral ureteral reimplantation according to Cohen: } \\
\text { 1(7.7\%) } \\
\text { - Kabul } \\
\text { - Peysterior urethral valves: } 1(7.7 \%) \\
\text { - Gastroschisis: } 1(7.7 \%)\end{array}$ \\
\hline Pre-operative condition & $\begin{array}{l}\text { O Ureteral DJ stent: } 6(33.3 \%) \\
\text { - Nephrostomy tube: } 2(11.1 \%) \\
\text { - Ureteral DJ stent + nephrostomy tube: } 2(11.1 \%)\end{array}$ \\
\hline Pre-operative hemogiobin (g/dL) median (IQR) & $13.2(12.1-13.7)$ \\
\hline Pre-operative creatinine (ng/dL) median $(\mathrm{QQR})$ & $0.7(0.5-1.1)$ \\
\hline Pre-operative sodium (mEq/dL) median (IQR) & $140(138-142)$ \\
\hline Pre-operative potassium (mEq/dla median (IQR) & $4.6(4.3-4.7)$ \\
\hline Pre-operative caician (mg/do $)$ median (IQR) & $9.8(9.6-9.9)$ \\
\hline Pre-operative uric acid (mg/di) median (IQR) & $3.9(3.4-4.2)$ \\
\hline Positive pre-operative urine culture (\%) & $\begin{array}{l}5.6(1 / 18) \\
\text { - Pseudomonas aeruginosa }\end{array}$ \\
\hline
\end{tabular}

* Cumulative stone size was evaluated as sum of largest stones diameter $\mathrm{BMI}=$ body mass index; IQR = inter-quartile range; $\mathrm{vmPCNL}=$ semi-closed-circuit vacuumassisted Mini-PCNL. 
Table 2. Intra- and post-operative data of the patients submitted to vmPCNL

\begin{tabular}{|c|c|}
\hline Parameters & Values \\
\hline Operative time (min) median (IQR) & $128(99-167)$ \\
\hline Tract location & $\begin{array}{l}\text { Upper calyx: } 3(12.5) \\
\text { Middle calyx: } 7(29.2) \\
\text { Lower calyx: } 14(58.3)\end{array}$ \\
\hline Tract & $\begin{array}{l}\text { Single: } 13(72.2) \\
\text { Multiple: } 5 \text { (27.8) }\end{array}$ \\
\hline Intra-operative complications (\%) & 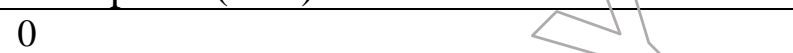 \\
\hline Exit strategy & $\begin{array}{l}\text { - Nephrostomy tube: } 15(83.3 \%) \text { [in one case: two } \\
\text { nephrostomy tubes] } \\
\text { - Tubeless: } 3(16.7 \%) \\
\text { - double-J ureteral stent: } 3(16.7 \%) \\
\text { - single-J ureteral catheter: } 5(27.8 \%)\end{array}$ \\
\hline Stone composition & $\begin{array}{l}\text { - Calcium oxalate monohydrate: } 3(2.31 \%) \\
\text { - Calcium oxalate monohydrate and cearicium } \\
\text { carbonate: } 3(23.1 \%) \\
\text { - Cysticie: } 2(15.4 \%) \\
\text { - Phosphate-based infectious stones (e.g. struvite, } \\
\text { carboapatite): } 5(38.5 \%)(\text { ) }\end{array}$ \\
\hline Fever $(\%)$ & $27.8(5 / 18)$ \\
\hline Blood transfusion $(\%)$ & $0<2$ \\
\hline $\begin{array}{l}\text { Complications according to Clavien-Dindo } \\
\text { classification }(\%) \\
\text { - Grade I-II } \\
\text { - Grade IIIa }\end{array}$ & $\begin{array}{l}-33.3(6,18) \\
-5.6(1 / 18)\end{array}$ \\
\hline Hospital stay (days) median (IQR) & $3(3-5)$ \\
\hline Post-operative hemoglobin (g/d $L)$ median (IQR)* & $(2.1)(11.3-13.2)$ \\
\hline Post-operative creatinine (mg/dL) median (IQR)** & $0.61(0.52-0.86)$ \\
\hline Post-operative CRP (mg/L/) median (IQR)** & $3.3(1.8-4.8)$ \\
\hline Stone-free rate $(\%)$ & $81.3(13 / 16)$ \\
\hline Stone-free rate after anciliary procedures $(\%)$ & $93.8(15 / 16)$ \\
\hline
\end{tabular}

vmPCNL = semi-ciosedi-circuit vacuum-assisted Mini-PCNL; CRP = C-reactive protein; IQR = interquartile range 
Table 3. Analysis of the disposable and reusable materials cost for mini-PCNL, stratified for miniPCNL using a reusable set (standard) and vacuum-assisted mini-PCNL (excluding endoscopes)

\begin{tabular}{|c|c|c|c|c|}
\hline Materials & Quantity & Price $(€)$ & $\begin{array}{c}\text { Standard } \\
\text { mini-PCNL }(€)\end{array}$ & $\begin{array}{c}\text { Vacuum-assisted } \\
\text { mini-PCNL }(€)\end{array}$ \\
\hline Ureteral catheter & 1 & 10.0 & 10.0 & 10.0 \\
\hline Hydrophilic wire & 2 & 24.3 & 48.6 & 48.6 \\
\hline Basket & 1 & 146.2 & 146.2 & 48.3 \\
\hline Laser fiber* & 1 & 888.2 & 88.8 & 88.8 \\
\hline Nephrostomy set & 1 & 73.2 & 73.2 & 73.2 \\
\hline Irrigation set** & 1 & 136.1 & 78.4 & 78.4 \\
\hline Contrast & 1 & 29.3 & 29.3 & 29.3 \\
\hline Lubrication & 2 & 1.9 & 3.8 & 3.8 \\
\hline Gowns/gloves & 5 & 2.5 & 12.7 & 12.7 \\
\hline Draping & 1 & 62 & $62.0(0$ & 62.0 \\
\hline Miscellaneous & I & 23 & 23.6 & 23.6 \\
\hline PCNL set*** & 1 & & 134.1 & l \\
\hline ClearPetra & 1 & 256.2 & I & 256.2 \\
\hline Total & 12 & 1 & 710.7 & 734.8 \\
\hline
\end{tabular}

* Depreciation calculated on 10 procedures

** Depreciation calculated on 4 procedures

***Depreciation calculated on the number of pediatric PCNLs performed in the last 24 months 


\section{TITLES OF FIGURES}

Figure 1. A) The ClearPetra® system (Well Lead Medical Co., Ltd., China), is shown. A plug is put over the external access to prevent the medium from flowing out. The sheath is equipped with a lateral arm connected to the aspiration system through a $200 \mathrm{ml}$ plastic bottle, which collects stone fragments. B) The pressure vent on the lateral arm is used to regulate aspiration and the red stripe on the sheath is the mark for endoscope retraction. D) Lapaxy is performed by slowly drawing back the nephroscope inside the sheath until the red stripe on the sheath, thus aspiring the fragments in the lateral arm.

Figure 2. Estimation of the pressures involved in the empioyed system. The entire circuit was considered as closed for estimation purposes. Bernoulli's principle states the total pressure is a constant in the system and derives from the sum of static and dynamic pressures. In the example shown, we considered the 16-Fr nephrostomy sheath and 12-Fr nephroseope, evaluating the aspiration pressure in $p=-13.013 \mathrm{~Pa}=-133 \mathrm{cmH}_{2} \mathrm{O}$. To estimate the presme with a 9.5-Fr ureteroscope and a 14-Fr nephrostomy sheath, the values of $r_{1}$ and $r_{2}$ were forodified accordingly, obtaining a final aspiration pressure $p=-14.00 \mathrm{~Pa}=-143 \mathrm{CmH}_{2} \mathrm{O}$. 


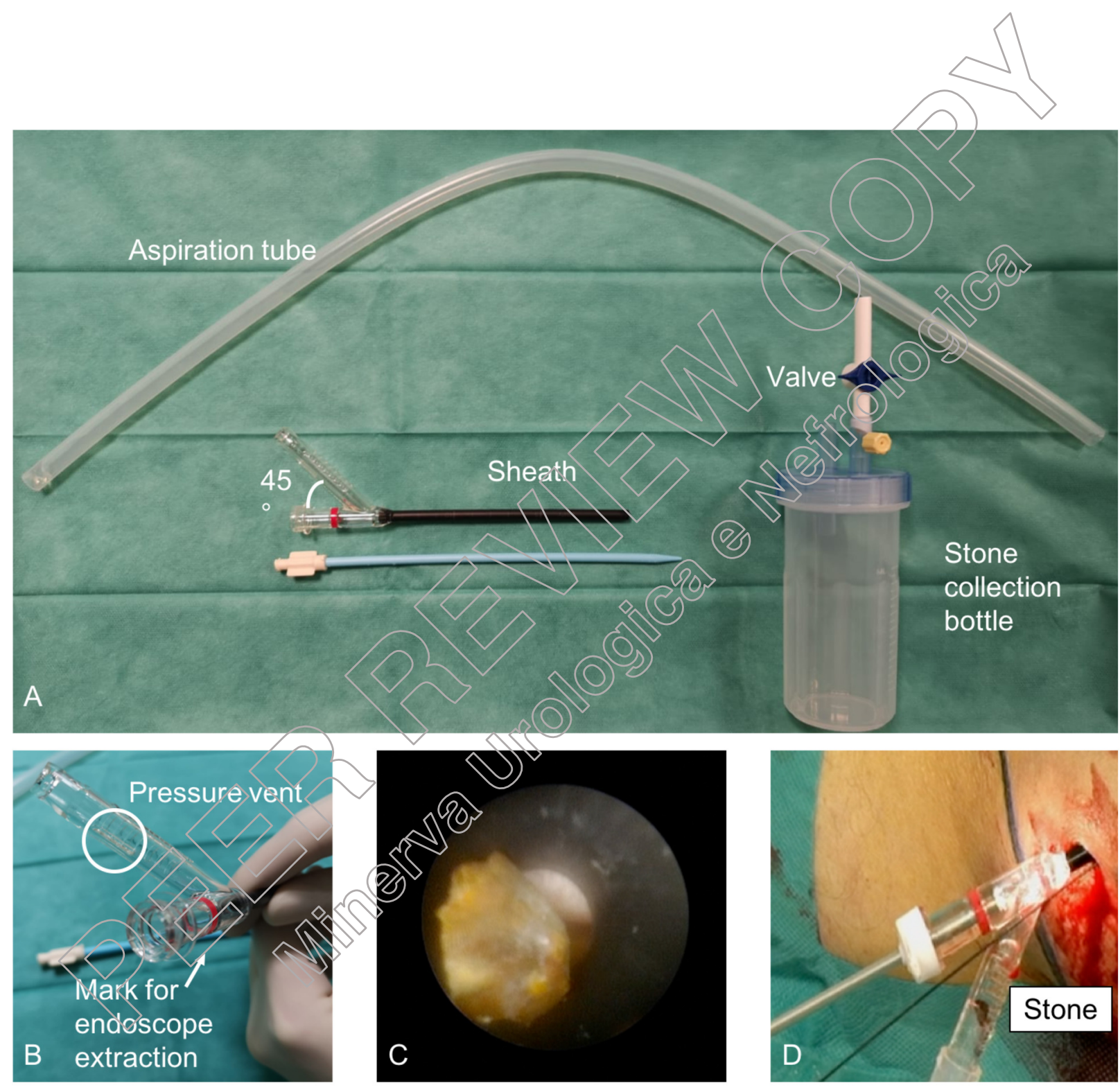




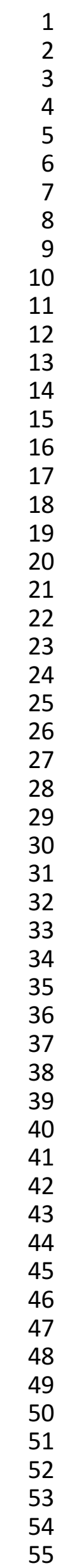




\section{Supplementary Digital Material}

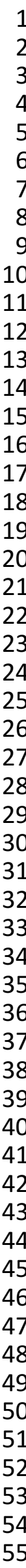

Download supplementary material file: Minerva Urol Nefrol-3951_Supplementary Digital Material1_V1_2020-05-15.docx

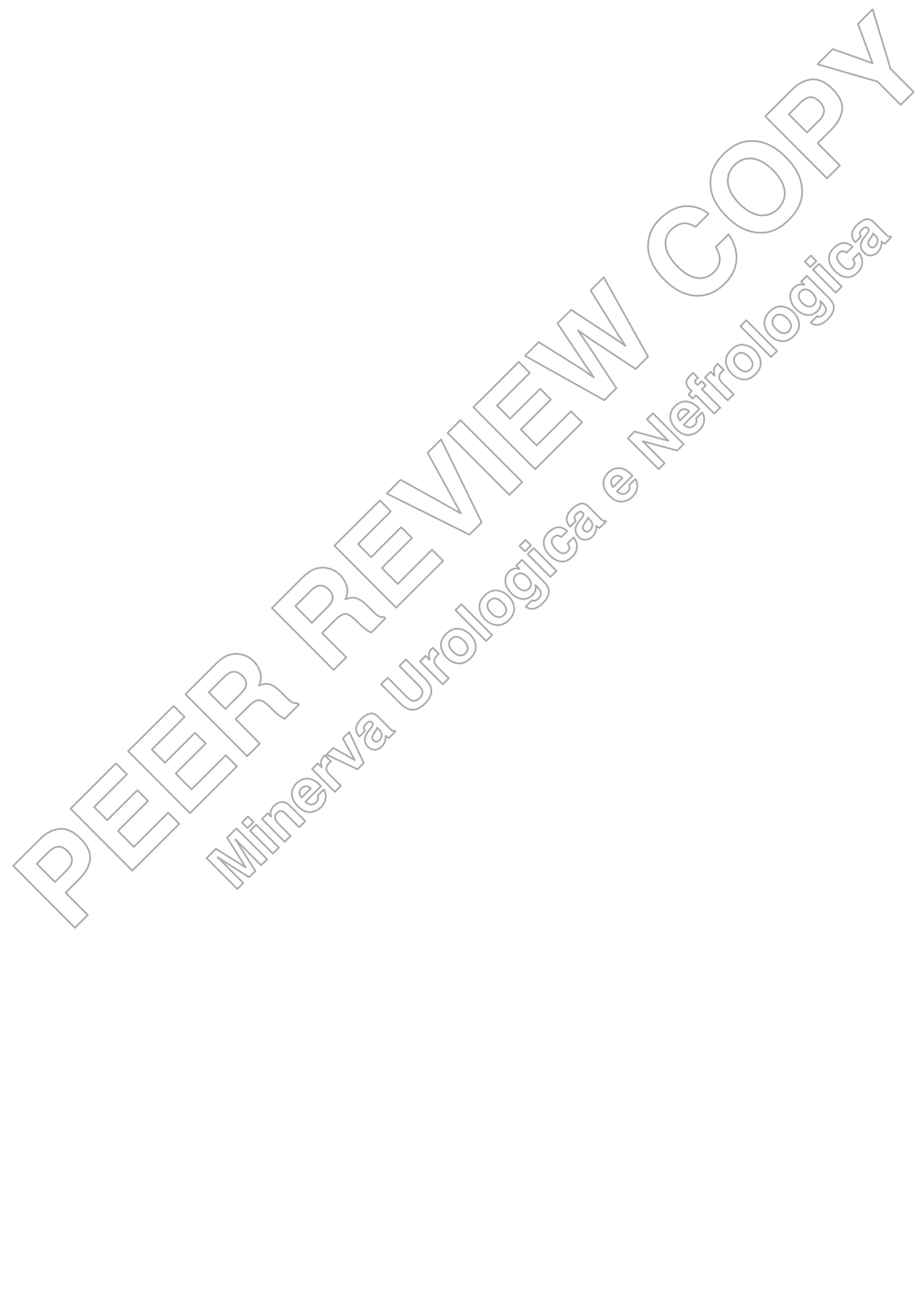

